\title{
Application of the density matrix renormalization group to the two level pairing model
}

\author{
J. Dukelsky ${ }^{1}$ and G. G. Dussel ${ }^{2}$ \\ ${ }^{1}$ Instituto de Estructura de la Materia, C.S.I.C., Serrano 123, 28006 Madrid, Spain \\ ${ }^{2}$ Departamento de Física “Juan Jose Giambiagi,"' Facultad de Ciencias Exactas y Naturales, Universidad de Buenos Aires, \\ Pabellón 1, Ciudad Universitaria, (1428) Buenos Aires, Argentina
}

(Received 24 February 1999)

\begin{abstract}
We introduce the density matrix renormalization group as an efficient truncation scheme which can be applied to nuclear shell model problems. To illustrate the power of the method we perform a calculation for the two level pairing model showing its convergence properties. [S0556-2813(99)50406-8]
\end{abstract}

PACS number(s): 21.60.Cs, 21.10.Pc, 21.30.Fe

Shell model exact diagonalization has been a powerful technique to describe low energy spectra in nuclei although limited, by the dimensions of the matrices involved, to the study of light nuclei. Quite recently the scope of the shell model has been extended to nuclei in the $f$ - $p$ shell [1]. The largest diagonalization performed so far has been done for ${ }^{52} \mathrm{Fe}$ [2] with a Hamiltonian matrix of dimension $10^{8}$ in $\mathrm{m}$ scheme. Future improvements, like including the use of the $j-j$ coupling scheme and quasispin formalism [3], are not expected to go beyond the $f-p$ shell in the near future due the enormous increase in memory required.

Shell model Monte Carlo methods [4] provide an alternative to go beyond the limits of direct diagonalization but they can predict only overall nuclear properties (masses, strength distributions, deformation, etc.). Another possible way to extend the applicability of the shell model, which we will introduce in this Rapid Communication, is to set up an efficient truncation scheme able to reduce significantly the shell model space such that the resultant dimensions are still tractable by actual computers. Such a technique, the density matrix renomalization group (DMRG), has recently been developed in the context of one-dimensional spin systems [5]. Since then it has been widely applied to low dimensional quantum lattice models like the Hubbard model, the $t-J$ model, the Holstein model, etc., with great success (for a review see [6]). The method is based on an iterative procedure which beginning from a small part of the system (block), whose dimensions are manageable, it then increases the block size step by step performing a truncation in each step such that the dimension of the block stays constant. The key point of the DMRG is the truncation method which allows us to retain the most important many body states that optimally represent the ground state and low lying excited states of the complete system.

The aim of this Rapid Communication is to introduce the DMRG in the framework of nuclear models and to exemplify its power in selecting the optimal states to be retained in each step of the procedure in a squematic two level pairing model (TLPM). Suppose that we want to obtain a reliable description of a system of size $M$. In its original derivation the DMRG was applied to a one-dimensional spin system, consequently $M$ had the meaning of the number of spin sites. For a finite Fermi system (like a nucleus) $M$ might be related to the number of single particle states $(\alpha j m)$. We first divide the system in three blocks like in Fig. 1, where each black dot corresponds to a single state ("site"). We assume to have a complete knowledge of the $m$ states of the left block $(L)$ of size $l$ ( $l$ is equal to 4 in the figure), and of the matrix elements of all relevant operators inside the block. We would like to increase the size of the block by one incorporating the central block $(C)$ with one site, but in doing so the dimension of the new enlarged block is doubled $(2 m)$. The aim of the DMRG method is to truncate the dimension of the new block retaining the "optimum" $m$ states and neglecting the other $m$ states. Subsequently the relevant operators are also truncated to this subspace of dimension $m$. In this way we increase the size of the block by one, while preserving the dimension $m$. The procedure is continued from left to right until the size of the left block exhausts the complete system finishing a sweep. For finite systems we may have to perform several sweeps from left to right and from right to left until we reach the desired precision.

Before going to a more detailed description of the method in the TLPM, we will show how to determine the "optimum" states in each step of the procedure. Suppose we are in an intermediate step in which the system is divided in the two blocks of Fig. 1. We have already incorporated the central block $(C)$ to the $L$ block to compose the enlarged block $L^{\prime}$ shown in Fig. 1 by the dashed line. The other block (right block $R$ ) of size $M-l-1$ plays the role of the environment. We will assume that we have a knowledge of the groundstate wave function of the entire system $|\Psi\rangle$. Let $|\alpha\rangle$ be the set of $2 m$ states of the $L^{\prime}$ block and $|\beta\rangle$ the set of states of the $R$ block. Expanding the ground-state wave function $|\Psi\rangle$ in the product space $L^{\prime} \times R,|\Psi\rangle=\Sigma_{\alpha \beta} \Psi_{\alpha \beta}|\alpha\rangle|\beta\rangle$ we are now able to define the reduced density matrix of the $L^{\prime}$ block

$$
\rho_{\alpha \alpha^{\prime}}=\sum_{\beta} \Psi_{\alpha \beta}^{*} \Psi_{\alpha^{\prime} \beta}
$$

which plays a central role in the DMRG method. We would like now to find a subset of $m$ states of the $L^{\prime}$ block $|p\rangle$

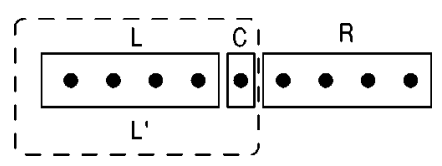

FIG. 1. Block separation of the system in the first iteration with $l=4$. $L^{\prime}$ represents the enlarged left block after the first renormalization step. 
$=\Sigma_{\alpha=1,2 m} \varphi_{\alpha}^{p}|\alpha\rangle$, such that the truncated wave function $|\bar{\Psi}\rangle$ $=\Sigma_{p, \beta} \bar{\Psi}_{p, \beta}|p\rangle|\beta\rangle$ optimally represents the ground-state wave function $|\Psi\rangle$, or in a more clear mathematical statement, "optimal" representation means maximization of the overlap $\langle\Psi \mid \bar{\Psi}\rangle$. The solution to this problem (for details see [7]) is given by a diagonalization of the reduced density matrix (1)

$$
\sum_{\alpha^{\prime}} \rho_{\alpha \alpha^{\prime}} \varphi_{\alpha^{\prime}}^{p}=\omega_{p} \varphi_{\alpha}^{p}
$$

The eigenvalues $\omega_{p}$ represent the probability of the system being in the state $|p\rangle$. Consequently, the "optimal" $m$ states of the $L^{\prime}$ block correspond to the $m$ highest eigenvalues $\omega$. Since the trace of the reduced density matrix is normalized to $1\left(\sum_{p=1}^{2 m} \omega_{p}=1\right)$ we can define the quantity $P_{m}$ $=\sum_{p=1}^{m} \omega_{p}$ such that $1-P_{m}$ gives an idea of the importance of the states we are neglecting.

If we want to obtain several excited low lying states from the DMRG procedure, we will have to construct at each step of the iteration a mixed state for the system built on several states of the superblock $\left|\Psi^{q}\right\rangle$. Assuming that we want to work out the DMRG procedure for $s$ states $(s-1$ excited states plus the ground state) the reduced density matrix will be of the form

$$
\rho_{\alpha \alpha^{\prime}}=\sum_{q=1}^{s} W_{q} \sum_{\beta} \Psi_{\alpha \beta}^{q *} \Psi_{\alpha^{\prime} \beta}^{q}
$$

Once the mixed reduced density matrix is defined in Eq. (3) the procedure follows in the same way as before, but by choosing equal mixing probabilities $W_{q}$ the lowest $s$ of the $m$ preserved block states in each step will represent optimally the $s$ targeted superblock states. Here obviously $m$ should be greater than $s$.

Having presented the general ideas of the DMRG method we will now proceed to apply it to the TLPM. The TLPM is a model of two orbits with degeneracy $\Omega=j+\frac{1}{2}$ and a single particle energy splitting $\varepsilon$. The pairing Hamiltonian in this model space is

$$
H=\frac{\varepsilon}{2} \sum_{\sigma} \sigma N_{\sigma}-G \Omega \sum_{\sigma \sigma^{\prime}} A_{\sigma^{\dagger}}^{\dagger} A_{\sigma^{\prime}}
$$

where $\sigma$ takes the values 1 for the upper level and -1 for the lower level. $N_{\sigma}$ and $A_{\sigma}^{\dagger}$ are the number and monopole pair operator of the level $\sigma$, respectively,

$$
\begin{gathered}
N_{\sigma}=\sum_{\mu=-j}^{j} a_{\sigma \mu}^{\dagger} a_{\sigma \mu}, \\
A_{\sigma}^{\dagger}=\frac{1}{\sqrt{\Omega}} \sum_{\mu>0}(-)^{j-\mu} a_{\sigma \mu}^{\dagger} a_{\sigma-\mu}^{\dagger},
\end{gathered}
$$

where $a_{\sigma m}^{\dagger}$ creates a particle in level $\sigma$ with $z$ angular momentum projection $\mu$.
For a system at half filling, the total number of particles $N$ equals half the total system degeneracy $(N=2 \Omega)$. The normalized states in the Hilbert subspace of the monopole pairs are

$$
|n\rangle=\frac{\Omega^{\frac{\Omega}{2}}}{\Omega !} A_{1}^{\dagger n} A_{-1}^{\dagger(\Omega-n)}|0\rangle, \quad 0 \leqslant n \leqslant \Omega .
$$

Here $n$ is the number of monopole pairs in the upper level.

The matrix Hamiltonian is a tridiagonal of dimension $\Omega$ +1 , with matrix elements

$$
\begin{gathered}
h_{n, n}=\langle n|H| n\rangle=\varepsilon(2 n-\Omega)-G\left(2 \Omega n-2 n^{2}+\Omega\right), \\
h_{n-1, n}=\langle n-1|H| n\rangle=-G n(\Omega-n+1) .
\end{gathered}
$$

The Hamiltonian (4) has two different phases depending on the adimensional parameter $x=2 G \Omega / \varepsilon$. For $x<1$ the system is characterized by pairing fluctuations on top of a Hartree-Fock reference state. The particle-particle selfconsistent RPA (SCRPA) [8] describes very well this region until its breakdown for $x \sim 1$. For $x>1$ the system becomes superfluid.

The DMRG method which we will apply here acts directly on the Hamiltonian matrix (7), dividing it into three blocks. The left block $L$ of size $l$, the central block $C$ with only one site, and the right block $R$ of size $(\Omega-l)$ sites. This procedure has some similarities with the one used by White and Noack [9] to study the tight-binding model in one dimension which was the precedent for the formal derivation of the DMRG [7]. The total system wave functions can be projected onto the subspaces of the three blocks as

$$
\Psi^{p}(i)=\left\{\begin{array}{rr}
L^{p}(i) & 1 \leqslant i \leqslant l \\
C^{p}(i) & i=l+1 \\
R^{p}(i) & l+2 \leqslant i \leqslant \Omega+1 .
\end{array}\right.
$$

Assuming that we want to obtain as a result of the renormalization procedure the $s$ lowest states of the system [including the ground state (g.s.)], we will have to diagonalize in each step a superblock Hamiltonian matrix of dimension $2 s+1$,

$$
H_{\mathrm{SB}}=\left(\begin{array}{lll}
H_{L} & H_{L C} & 0 \\
H_{C L} & h_{l+1, l+1} & H_{C R} \\
0 & H_{R C} & H_{R}
\end{array}\right),
$$

where $H_{L}$ is an $s \times s$ matrix with the matrix elements of the Hamiltonian between the states $L^{p}(1 \leqslant p \leqslant s), H_{C L}$ is a vector of dimension $s$ containing the interaction between site $l$ +1 and the block $L$ for each of the $s$ states, $H_{R}$ is an $s \times s$ matrix with the matrix elements of the Hamiltonian in the block $R$, and $H_{C R}$ is a vector of dimension $s$ containing the interaction between site $l+1$ and the block $R$ for each of the $s$ states.

From the $2 s+1$ eigenvectors of the superblock matrix $H_{\mathrm{SB}}$ we retain the lowest $s$ eigenvectors $\varphi^{p}$. Since in this simple model the wave functions are unvalued we only need to project the $s$ superblock eigenvectors onto the space $L^{\prime}$ of the first two blocks ( $L$ and $C$ for a sweep from left to right, 
see Fig. 1) of dimension $s+1$ instead of following the more general procedure outlined before for general many body wave functions:

$$
\phi_{\alpha}^{p}=\frac{\varphi_{\alpha}^{p}}{\sqrt{\sum_{\alpha=1}^{m+1}\left(\varphi_{\alpha}^{p}\right)^{2}}}, \quad \alpha=1, \ldots, m+1
$$

In order to continue the procedure we have to neglect one state. Note that in general we will have to disregard half of the states. Following White we construct a reduced density matrix $\rho$ in the $(s+1)$-dimensional subspace mixing the $s$ wave function (8) with probability $W_{k}$,

$$
\rho_{p q}=\sum_{k=1}^{s} W_{k} \phi_{p}^{k} \phi_{q}^{k} \text {. }
$$

To ensure that the $s$ states are equally represented in the mixed density matrix (9) we assume equal probabilities $W_{k}$ $=1 / \mathrm{s}$. We next diagonalize the density matrix

$$
\sum_{q} \rho_{p q} \chi_{q}^{n}=\omega_{n} \chi_{p}^{n}
$$

The eigenvalues $\omega_{n}$ of the density matrix represent the probability of finding the corresponding $\chi^{n}$ eigenvector in the mixed state of the total system. Consequently we disregard the eigenvector corresponding to the lowest eigenvalue and then we proceed to renormalize the wave function and Hamiltonian matrix of the new block $L^{\prime}$ with $l+1$ sites,

$$
\begin{gathered}
L_{i}^{n}(l+1)=\sum_{p=1}^{s} L_{i}^{p}(l) \chi_{p}^{n} \quad \text { for } \quad i \leqslant l \quad \text { and } n \leqslant s \\
L_{l+1}^{n}(l)=\chi_{s+1}^{n}, \\
H_{L}(l+1)_{p q}=\sum_{r, s=1}^{s} H_{L^{\prime}}(l+1)_{r s} \chi_{r}^{p} \chi_{s}^{q}
\end{gathered}
$$

where

$$
H_{L^{\prime}}(L+1)=\left(\begin{array}{rr}
H_{L} & H_{L C} \\
H_{C L} & h_{l+1, l+1}
\end{array}\right) .
$$

The renormalization procedure begins with a warm up in which we construct the first guesses for the wave functions $L$ and the Hamiltonians $H_{L}$. We first diagonalize a $(2 s+1)$ dimensional superblock matrix constructed with the bare Hamiltonian in the first $2 s+1$ sites. With the $s$ lowest superblock eigenvectors projected as in Eq. (8) we obtain the density matrix $\rho$ (9) of dimension $s+1$. With the highest $s$ eigenvectors we renormalize the wave function $L(11)$ and the Hamiltonian (12) for the augmented block of size $l=s$ +1 and then proceed to the next site to the right always using $s$ sites for the right block $R$. The warm up finishes when we arrive at the position $\Omega-s$ having now a first representation for the wave functions $L$ and the Hamiltonians $H_{L}$. In the next sweep we go from right to left constructing the wave function $R$ and the Hamiltonian matrices $H_{R}$ using

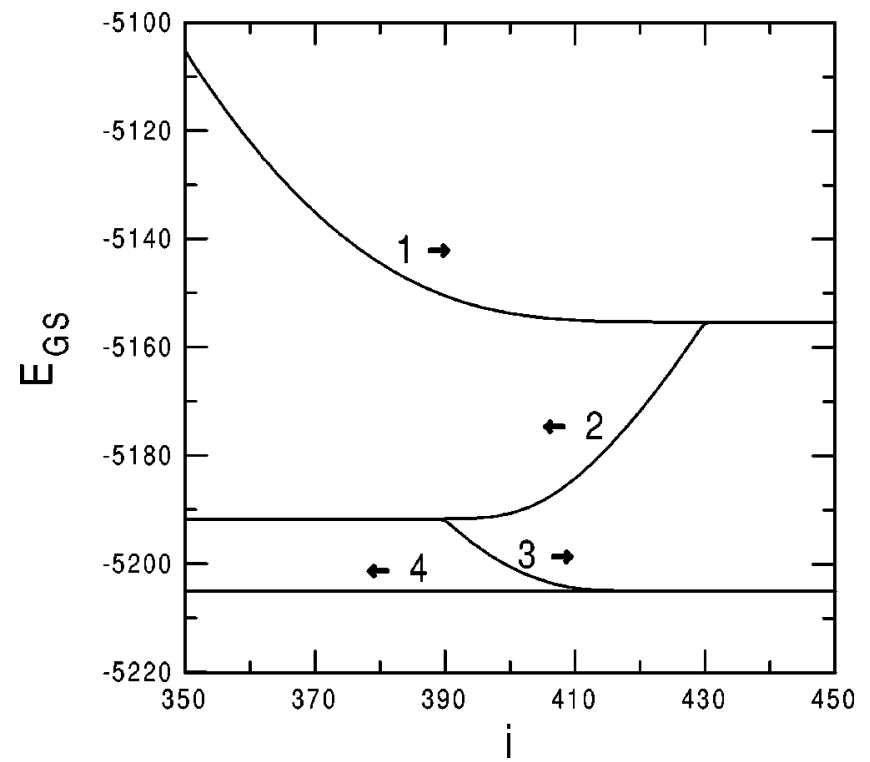

FIG. 2. Ground-state energy of the TLPM with $\Omega=1000, \varepsilon$ $=2$, and $x=5$ for each sweep as a function of the renormalization step (site).

the already renormalized wave functions $L$ and the Hamiltonian matrices $H_{L}$ of the previous sweep as the environment. In each sweep we improve the representation of either left or right wave functions and Hamiltonian matrices. At the end of each sweep we compare the $s$ energies with those of the previous sweep to check convergence. The procedure continues from right to left and from left to right until we reach the desired precision.

In order to illustrate the power of the DMRG method we have applied it to a TLPM with $\Omega=1000, \varepsilon=2$, and $x=5$, corresponding to a well established superconducting phase. We demand the DMRG method to provide the ground state plus three excited states implying that $s=4$. In the DMRG procedure we will have to obtain iteratively the lowest four eigenstates of a $9 \times 9$ superblock matrix of Eq. (8) and then completely diagonalize the density matrix (9) of dimension $5 \times 5$. Here the advantages of the DMRG are clearly seen, we have to diagonalize iteratively matrices of dimensions 9 and 5 as compared to a large scale diagonalization of dimension 1001 in this simple case. After each sweep $j$ is finished we calculate the relative mean deviation energy $\sigma_{E}^{j}$ of the four states compared with those of the previous sweep,

$$
\sigma_{E}^{j}=\sum_{p=1}^{4} \frac{\left|E_{p}^{j}-E_{p}^{j-1}\right|}{\left|E_{p}^{j-1}\right|} \text {. }
$$

The iteration is stopped if $\sigma_{E}^{j} \leqslant 10^{-10}$, which for the present system requires five sweeps.

In Figure 2 we show the convergence of the g.s. energy in each iteration of the five sweeps, the horizontal axis corresponds to the sites. Odd sweeps go from left to right improving the description of the left blocks while even sweeps go from right to left improving the right blocks, as indicated by the arrows. The energy in the fifth sweep is indistinguishable from that of the fourth sweep. The figure is restricted to the portion between site 350 and site 450 in which there is an appreciable variation of the energy after the first sweep. This 


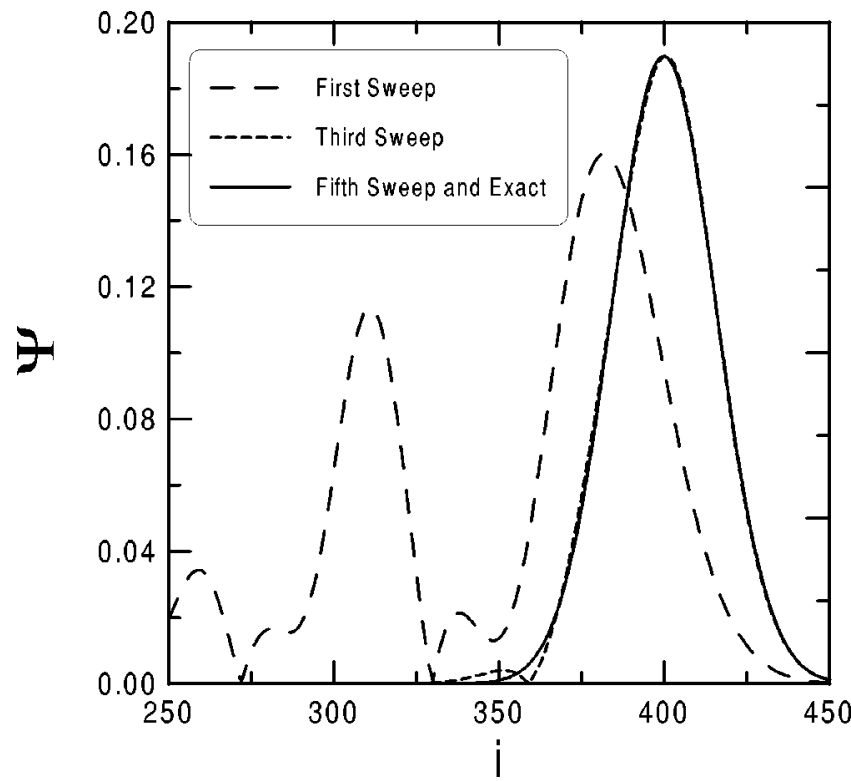

FIG. 3. Ground-state wave functions at the end of the left to right sweeps in the region $250<i<450$.

region in which the energy is modified along the sweep is directly correlated with the extension of the wave function. The figure also shows the variational character of the DMRG [10], the energy is continuously improved until the convergence point. The convergence of the three excited states included in the calculation follows the same pattern as the convergence of the g.s. displayed in the figure.

In Fig. 3 we show the g.s. wave function obtained in the first, third, and fifth final sweep. The latter coincides with the exactly diagonalized wave function with a precision of $10^{-9}$. At the end of the first sweep, due to the poor description of the environment (right block $R$ ) and the use of a mixed density matrix, the approximated ground-state wave function is strongly mixed with the excited states. As seen in the figure, the situation is dramatically improved after the second sweep in which the warm up for the left and right blocks is finished.

We have restricted here to a relative small degeneracy $\Omega=1000$ in order to exemplify the convergence properties of the DMRG method. Larger systems can be treated within the same procedure at the cost of using more CPU time in each sweep, but the memory requirements and the CPU time increases linearly with $\Omega$.

In summary, we have introduced the DMRG as a potentially useful truncation scheme to treat shell model problems beyond the capabilities of large scale diagonalizations. We have applied the method to the TLPM to demonstrate how the procedure works, improving at each step of the iteration the wave functions, the Hamiltonian matrix, and any other operator matrix desired. Though the DMRG method is firmly established for spin and fermion systems in one dimension and for small clusters in two dimensions, more work is still needed to optimize the method for general nuclear shell model calculations. Once this is done we believe that the method would be able to accurately describe the low lying states of medium and eventually heavy nuclei far beyond the actual limits of large scale diagonalizations.

We would like to thank M. A. Martin-Delgado and G. Sierra for helpfull discussions. J.D. acknowledges support from the DIGICYT under Contract No. PB95/0123. G.G.D. has been supported in part by PID No. 4547/96 of the CONICET, Argentina, PMT-PICT1855 of ANPCYT and Grant No. Ex-055 from the University of Buenos Aires.
[1] E. Caurier, A. P. Zuker, A. Poves, and G. Martinez-Pinedo, Phys. Rev. C 50, 225 (1994).

[2] C. A. Ur et al., Phys. Rev. C 58, 3163 (1998).

[3] E. Caurier, G. Martinez-Pinedo, F. Nowacki, A. Poves, J. Retamosa, and A. P. Zuker, Phys. Rev. C 59, 2033 (1999).

[4] S. E. Koonin, D. J. Dean, and K. Langanke, Phys. Rep. 278, 1 (1997).

[5] Steven R. White, Phys. Rev. Lett. 69, 2863 (1992).

[6] Steven. R. White, Phys. Rep. 301, 187 (1998).
[7] Steven R. White, Phys. Rev. B 48, 10345 (1993).

[8] J. Dukelsky, G. Röpke, and P. Schuck, Nucl. Phys. A626, 17 (1998).

[9] S. R. White and R. M. Noack, Phys. Rev. Lett. 68, 3487 (1992).

[10] S. Östlund and S. Rommer, Phys. Rev. Lett. 75, 3537 (1995); J. Dukelsky, M. A. Martín-Delgado, T. Nishino, and G. Sierra, Europhys. Lett. 43, 457 (1998). 